\title{
Numerical Simulation of the Dwarf Companions of Giant Galaxies
}

\author{
A. H. Nelson ${ }^{1}$ and P. R. Williams ${ }^{1}$ \\ ${ }^{1}$ School of Physics \& Astronomy, Cardiff University, Cardiff, UK \\ email: nelsona@cf.ac.uk
}

\begin{abstract}
We report simulations of the formation of a giant disc galaxy from cosmological initial conditions. Two sets of initial conditions are used, initially smooth density for both gas and stars, representing the Warm dark Matter scenario, and an initially fluctuating density representing the standard spectrum for the Cold dark Matter scenario. For the WDM initial conditions, the galaxy has a population of long lived dwarf satellites at $\mathrm{z}=0$, with orbits close to a plane coincident with that of the giant galaxy disc. The detailed properties of these dwarfs mimic closely the observed properties of Local Group dwarfs with respect to mass, and kinematics. However they do not have individual dark matter halos, but orbit in the nearly spherical dark matter halo of the giant galaxy. The reason for this is that the initial population of dwarf dark matter haloes, which form during the initial collapse phase, all merge into the halo of the giant galaxy within a few to several Gyears, while the long lived dwarfs form as a secondary population by gravitational collapse of high angular momentum gas in the outer reaches of the giants proto-galactic disc. Due to their late formation and their more distant orbits, they survive until the present epoch as individual dwarf galaxies at radii $20-50 \mathrm{kpc}$ from the giants centre. For CDM initial conditions there are many more dwarf satellites at $\mathrm{z}=0$, some of which form early on as gas condensations in DM sub-halos, and survive with these individual DM halos till $\mathrm{z}=0$ due to their being sufficiently well bound to avoid merging with the main galaxy. However even in this case some second generation satellites form as initially gas only objects, just as for the smooth initial conditions of WDM.
\end{abstract}

Keywords. Simulations, Galaxies, Dwarfs.

\section{Introduction}

The aim of our simulations is to investigate the detailed development of structure at the level of individual galaxies during their formation with as realistic gas physics as possible, and hence we concentrate all our computational resources on a section of the primordial medium with sufficient mass to form a single giant galaxy. These calculations have shown that the formation of detailed structures such as the gas and stellar disc, bars, spiral structure, stellar bulges and warps are only weakly dependent on the initial configuration of the mass, but depend mainly on the total mass, angular momentum and radius at initial collapse (when the Hubble expansion is reversed due to self gravity). This is because the violent relaxation which occurs during the period of maximum collapse and the subsequent fragmentation and remerging events lead to the initial configuration of the mass being forgotten (Williams \& Nelson 2001). This is in contrast to the situation for large scale structure calculations, where on a large scale the mass distribution has not departed very far from its initial configuration, and consequently the initial conditions are important in determining the final outcome.

While this holds for the central stellar and gas discs of giant galaxies, it turns out not be the case for any population of dwarf satellite galaxies that they may have. The 

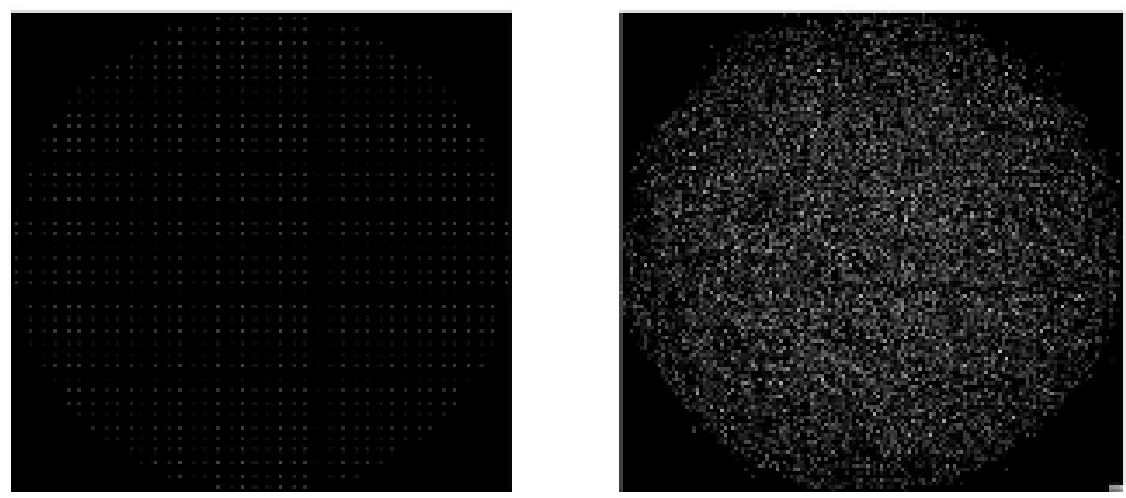

Figure 1. Initial Gas distribution (looking down rotation axis) (a) WDM on the left, (b) CDM on the right

results presented here indicate that the nature and number of such satellite galaxies does depend on the initial configuration of mass.

\section{Details of Simulations}

The features of the code used for the simulations include :- Tree-Code/SPH [ with $1 /(\mathrm{r}+\epsilon)$ potential ], Parallelised using MPI, Individual Particle Timesteps, Fully dynamic Kernel Radius, Star Formation via a Schmidt Law, Full Energy Equation with cooling and SN feedback. For details see Williams \& Nelson (2001).

The feedback method used follows the algorithm outlined by Springel (2000), except that we use a different formula for the decay of turbulent energy to thermal energy, based on the physics of the Kolmogoroff cascade, i.e. dq/dt is proportinal to $q^{3 / 2}$, where $q$ is the specific energy density in turbulent gas energy generated by supernovae.

Initial Conditions:- Total Mass $5 \times 10^{11} M_{\odot}(90 \%$ non-baryonic DM, $10 \%$ baryonic gas), Initial Radius $=175 \mathrm{kpc}$, initially in Hubble expansion with an overdensity of 1.6 at $\mathrm{z}=4$, initially solid body rotation with angular velocity $=0.25$ rads./ Gyr.

We consider two cosmic fluctuation cases :- Warm Dark Matter - Fig 1a (where we assume that the supression of small scale fluctuations makes the dark matter and gas initially smooth on the scale of $175 \mathrm{kpc}$ ), and Cold Dark Matter - Fig 1b (where the standard fluctuation spectrum (Efstathiou et al. 1992) is applied at $\mathrm{z}=4$ with amplitude scaled to $\left.\sigma_{8}=0.9\right)$.

\section{Results}

Fig 2 Shows the final state of the WDM initial conditions at $\mathrm{z}=0$. The top panels show the gas, stars and dark matter face-on, while the botttom panels show the edge-on view. The central galaxy has a small bar, and weak spiral arms in the gas disk, with 5 satellite galaxies distinguishable at $\mathrm{z}=0$. The satellites are identified as gas density maxima with a group of gravitationally bound gas particles around them. Their internal structure is not resolved, but they have physical integrity in that they are long lived and support themselves against further collapse by rotation.

Their properties in order of total mass (in $10^{6} M_{\odot}$ ) are summarised in Table 1 . All of these final satellites form late at around $\mathrm{z}=0.2$ and persist to the end. None have a bound dark matter component. 

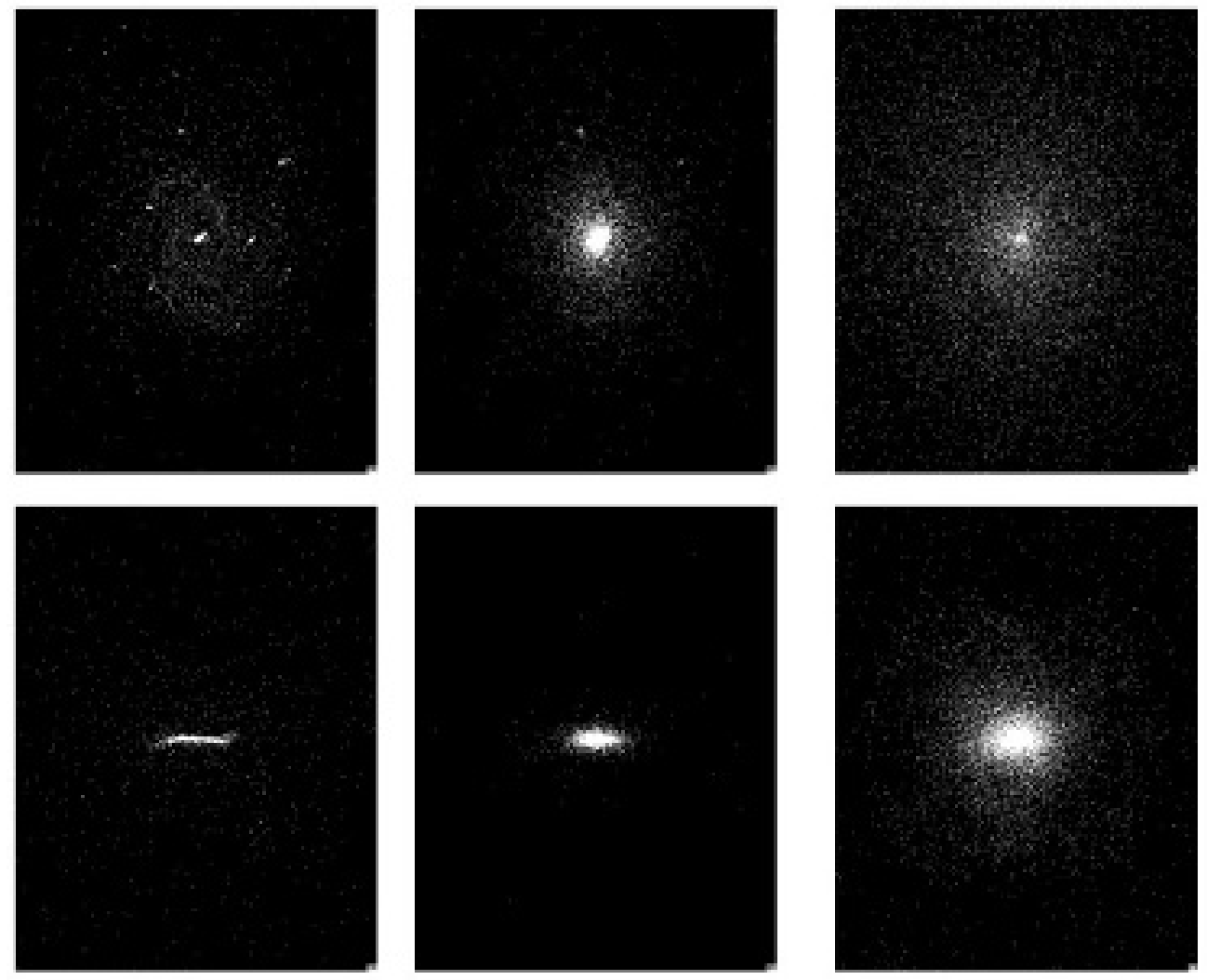

Figure 2. WDM final state at $\mathrm{z}=0$

Table 1. Properties of Satellites at $z=0$ for WDM

\begin{tabular}{rcrccc}
\hline Gas mass & dark mass & star mass & total mass & Size $(\mathrm{kpc})$ & velocity \\
\hline 125.4 & 0.0 & 45.0 & 170.4 & 0.9 & 6.6 \\
106.3 & 0.0 & 10.0 & 116.3 & 1.1 & 5.9 \\
95.6 & 0.0 & 17.5 & 113.1 & 1.6 & 7.2 \\
85.8 & 0.0 & 5.0 & 90.8 & 1.6 & 4.1 \\
90.5 & 0.0 & 0.0 & 90.5 & 1.8 & 10.8 \\
\hline
\end{tabular}

Fig 3 Shows the final state of the CDM initial conditions at $z=0$. Again The central galaxy has a small bar, and weak spiral arms in the gas disk, with this time 18 satellite galaxies are distinguishable at $\mathrm{z}=0$.

Their properties in order of total mass (in $10^{6} M_{\odot}$ ) are summarised in Table 2 .

In this case 12 of the initial dark matter subhalos are sufficiently well bound to avoid merging, and survive to the end of the simulation. But at the same time 6 second generation satellites form as gas only objects around $\mathrm{z}=0.2$, just as for the smooth initial conditions of WDM. These are the satellites with zero dark mass.

One of the initial halos, which persists as a satellite to the end, has a retrograde orbit relative to the rotation of the galaxy disc, while some of the initial halos have high latitude orbits, well above the galactic plane. This contrasts with the WDM case where the final satellite orbits are all prograde, and are all close to the galactic plane. 

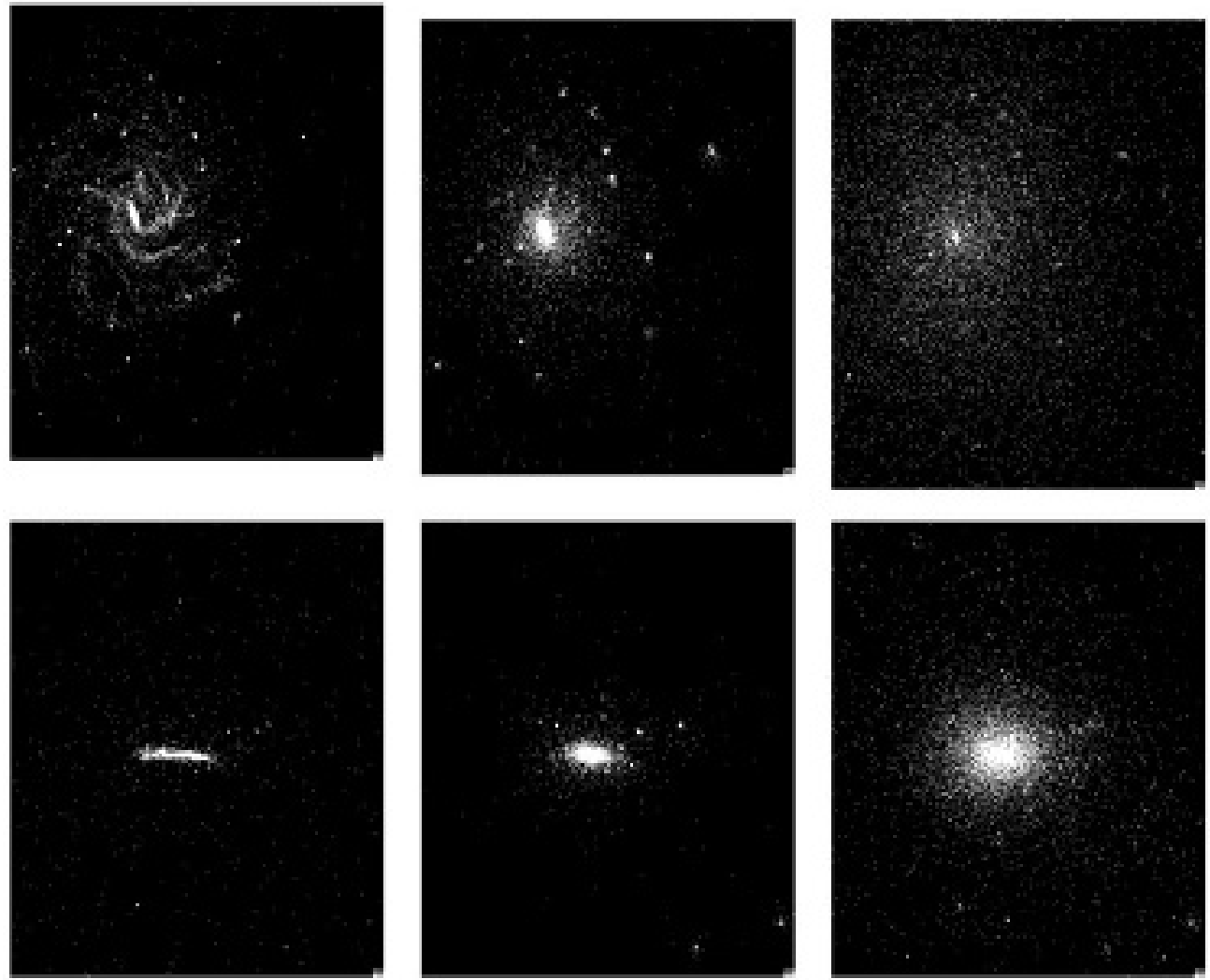

Figure 3. CDM final state at $\mathrm{z}=0$

Table 2. Properties of Satellites at $\mathrm{z}=0$ for CDM

\begin{tabular}{rrrrcc}
\hline Gas mass & dark mass & star mass & total mass & Size $(\mathrm{kpc})$ & velocity \\
\hline 42.5 & 352.6 & 262.8 & 657.9 & 0.8 & 20.6 \\
56.3 & 352.6 & 82.6 & 491.5 & 0.6 & 22.1 \\
21.1 & 286.5 & 70.1 & 377.7 & 0.9 & 15.3 \\
13.7 & 154.3 & 167.7 & 335.7 & 1.2 & 10.6 \\
43.1 & 154.3 & 55.0 & 252.4 & 1.0 & 10.9 \\
225.7 & 0.0 & 10.0 & 235.7 & 0.8 & 11.2 \\
42.5 & 176.3 & 15.0 & 233.8 & 0.7 & 8.3 \\
22.7 & 176.3 & 15.0 & 214.0 & 1.2 & 10.1 \\
27.9 & 154.3 & 20.0 & 202.2 & 23.4 & 6.6 \\
141.2 & 0.0 & 55.0 & 196.2 & 0.9 & 8.5 \\
18.9 & 88.2 & 37.5 & 144.6 & 1.0 & 7.5 \\
86.1 & 0.0 & 52.5 & 138.6 & 1.1 & 4.2 \\
25.2 & 110.2 & 2.5 & 137.9 & 3.6 & 6.6 \\
40.2 & 88.2 & 7.5 & 135.9 & 9.1 & 4.9 \\
107.2 & 0.0 & 0.0 & 107.2 & 1.1 & 4.6 \\
100.9 & 0.0 & 5.0 & 105.9 & 2.2 & 3.6 \\
33.2 & 44.1 & 5.0 & 82.2 & 7.5 & 2.6 \\
81.1 & 0.0 & 0.0 & 81.1 & 2.9 & \\
\hline
\end{tabular}




\section{Conclusions}

Some of the dwarf companions of giant galaxies may have formed recently with no non-Baryonic DM component. They would be gas rich with kinematics similar to Local Group dwarfs, raising the possibility of baryonic "dark matter" which could be in the form of molecular hydrogen (Lisenfeld et al. 2002, Taylor et al. 1999, Taylor \& Klein 2001,Madden et al. 1997, Papadopoulos 2002, Braine et al. 2001, Elmegreen \& Hunter 2000).

Smooth pre-cursor galaxy clouds produce low latitude, prograde orbits in satellites. While inhomogeneous pre-cursor galaxy clouds with sufficient fluctation amplitude to produce long-lived dark matter subhalos will have some high latitude and retrograde orbits in satellites.

\section{References}

Braine, J., Duc, P.-A., Lisenfeld, U., Charmandaris, V., Vallejo, O. Leon, S. \& Brinks, E. 2001, $A \mathscr{S A} 378,51$

Efstathiou, G., Bond, J. R. \& White, S. D. M. 1992, MNRAS 258, 1

Elmegreen, B. \& Hunter, D. A. 2000, ApJ 540, 814

Lisenfeld, U., Israel, F. P., Stil, J. M. \& Sievers, A. 2002, A\& A 382, 860

Madden, S. C., Poglitsch, A., Geis, N., Stacey, G. J. \& Townes, C. H. 1997, ApJ 483, 200

Papadopoulos, P. P. 2002, ApJ 579, 270

Springel, V. 2000, MNRAS 312, 859

Taylor, C. L., Huttemeister, S., Klein, U. \& Greve, A. 1999, A\&A 349, 424

Taylor, C. L. \& Klein, U. 2001, A\& $A$ 366, 811

Williams, P. R. \& Nelson, A. H. 2001, A\& $A$ 374, 839 